\title{
CONSTRUCCIÓN, VALIDEZ DE APARIENCIA Y CONFIABILIDAD DE UN CUESTIONARIO PARA EVALUAR LA CENTRAL DE ESTERILIZACIÓN
}

\author{
Mallerly Carolina Garzón Martínez*
}

\section{Resumen}

Introducción: la central de esterilización es una de las áreas más importantes en el control de infecciones de un hospital, debe ofrecer óptimas condiciones de calidad y regirse por un manual de buenas prácticas en esterilización que garantice la calidad de la atención y regule las actividades que allí se desarrollan. Objetivo: construir, validar la apariencia, confiabilidad y aplicar un cuestionario para evaluar las condiciones de la central de esterilización. Resultados: se construyó un cuestionario mediante consenso de expertos compuesto por 470 ítems subdivididos en tres capítulos: procesos en áreas, métodos de esterilización y garantía de la calidad. Dos observadores lo aplicaron en cuatro centrales de esterilización de Bogotá DC. Se estableció el acuerdo interobservador por medio del índice de Kappa obteniendo en la institución uno un índice de 0,76 , en las dos 0,91 , en tres 0,93 y en la cuatro 0,98 . Conclusión: el índice de Kappa final de 0.98 demuestra que hay muy buena concordancia entre las observaciones realizadas con el cuestionario, por lo tanto se puede establecer que este es un instrumento válido y confiable para evaluar las condiciones de la $\mathrm{CE}$.

Palabras clave: central, esterilización, cuestionario, evaluación, calidad.

Abreviaturas: CE, central de esterilización;

\section{DETERMINING THE APPEARANCE OF VALIDITY AND RELIABILITY OF AN EVALUATION QUESTIONNAIRE APPLIED IN STERILIZATION CENTERS}

\begin{abstract}
Introduction: the sterilization center is one of the most important areas for infection prevention in a hospital. It must offer optimal quality conditions and must implement best practices through a sterilization manual which ensures quality of care and regulates all activities. Objective:todeterminethe appearance of validity and reliability of an evaluation questionnaire applied to sterilization centers. Results: a consensus of experts designed a questionnaire including 470 items subdivided into three chapters: area procedures, sterilization methods and quality assurance. Two observers applied the questionnaire in four sterilization centers in Bogotá DC. Interobserver agreement was reported as a kappa index obtaining an index of 0.76 in institution one, 0.91 in institution two, 0.93 in institution three and 0.98 in institution four. Conclusions: the final kappa index of 0.98 demonstrates a high agreement between observers when this questionnaire is applied, thus, it can be determined that this is a valid and reliable instrument for evaluating sterilization centers.
\end{abstract}

Key words: center, sterilization, questionnaire, evaluation, quality.

Fecha recibido: mayo 4 de 2012 - Fecha aceptado: junio 29 de 2012

* Instrumentadora Quirúrgica, especialista en docencia universitaria, Profesor Asistente, Fundación Universitaria de Ciencias de la Salud, Bogotá DC. Colombia.
** Liceth Villamizar, Carlos Castro MD, Lizeth Beltrán:Asesores metodológicos División de Investigaciones, Fundación Universitaria de Ciencias de la Salud, Bogotá DC, Colombia. 


\section{Introducción}

La CE es un servicio que recibe, acondiciona, procesa, controla y distribuye ${ }^{1}$ los insumos medicoquirúrgicos y material (apósitos, gasas, mechas, cotonoides etc.), no solo del área quirúrgica sino del resto del hospital. El suministro de estos elementos exige procedimientos de baja, media y alta complejidad Además la CE debe velar por la calidad en los procesos de desinfección de todos los servicios de la institución. Es una sección de alto impacto en el control de las infecciones adquiridas en el hospital y sobre esta recae la responsabilidad del procesamiento de los insumos; si ocurren fallas, el resultado final de los dispositivos médicos no serán satisfactorios y por lo tanto repercute en la calidad del servicio prestado al paciente.

Hoy día la CE se concibe con un concepto diferente, como una subempresa dentro de las instituciones de salud. Los bienes y servicios que provee no solo son de tipo económico, sino que además deben ser seguros y de alta calidad. Esto ha llevado a la necesidad de centralizar los procesos, realizando todas las etapas de esterilización como son la limpieza, descontaminación, inspección, preparación, empaque, esterilización, almacenamiento y distribución, en un mismo recinto físico. Este sistema se considera más eficiente y seguro, debido a que existe supervisión por parte de una persona calificada, con criterios uniformes y manejo racional de los recursos.

El manual único de estándares y de verificación del sistema de habilitación ${ }^{2}$, refiere que la CE debe estar a cargo de un instrumentador quirúrgico o enfermera profesional y en aquellos lugares en que no se pueda contar con el personal mencionado, lo hará una auxiliar en enfermería. El personal contratado debe ser bien seleccionado, capacitado y evaluado en forma periódica, ya que de él depende en gran medida la garantía de calidad de los procesos.

En Colombia se estableció el Manual de Buenas Prácticas de Esterilización Resolución 02183 de 2002, como una herramienta del Sistema Obligatorio de Garantía de Calidad de la Atención en Salud (SOGC), en donde están descritas las normas básicas de los proce- sos y actividades de la $\mathrm{CE}^{3}$, su implementación está reglamentada en el sistema único de habilitación de las instituciones prestadoras de servicios de salud. ${ }^{4}$ Existen documentos de apoyo en donde se hacen esfuerzos orientados a la vigilancia, prevención y control de las infecciones intrahospitalarias, con el fin de mejorar la calidad de la prestación del servicio por medio de las CE. Aunque en Colombia se cuenta con normas, procedimientos y recomendaciones, es de gran importancia que las instituciones hospitalarias y los entes de vigilancia y control implementen herramientas de evaluación de cada uno de los procesos y actividades, orientados a garantizar la calidad de todos los insumos. Lo anterior exige la necesidad de diseñar un instrumento que permita evaluar y verificar las condiciones de la $\mathrm{CE}$ en todos los aspectos. El objetivo del trabajo es construir, validad la apariencia, confiabilidad y aplicar un cuestionario para evaluar las condiciones de la CE.

\section{Materiales y métodos}

El tipo de diseño consiste en la validez de apariencia y confiabilidad de un cuestionario para evaluar las condiciones de la CE. La investigación se desarrolló en tres etapas:

Etapa I: para la construcción el cuestionario se convocó un grupo de cuatro expertos en $\mathrm{CE}$, a quienes se les entregó un manual para la realización de los ítems y la estructura de los mismos. Se construyeron en forma individual basados en la normatividad vigente, la literatura existente y su experiencia en la coordinación de CE. Evaluaron de manera individual la pertinencia y relevancia de cada ítem, después se realizaron tres reuniones para el consenso de expertos, dando como resultado un cuestionario compuesto por 470 ítems.

Etapa II: dos observadores aplicaron el cuestionario construido por los expertos en la CE de la institución 1. Los datos fueron tabulados en Excel, utilizando el paquete estadístico Stata versión 10.0, luego se estableció el acuerdo interobservador por medio del índice de concordancia Kappa de Cohen. Analizados los ítems que presentaron desacuerdos se realizaron ajustes en su 
redacción. Se calculó el índice de concordancia Kappa (valor $\mathrm{K}$ ) entre los dos observadores de acuerdo con la siguiente escala ${ }^{5}$ de la Tabla 1.

Etapa III: los dos observadores aplicaron el cuestionario en tres CE de instituciones de cuarto nivel de la ciudad de Bogotá DC. Después se estableció el valor $\mathrm{K}$, se analizaron los ítems que presentaron desacuerdos y se realizaron los ajustes pertinentes.

\section{Resultados}

Etapa I: se elaboró un cuestionario con 470 ítems para evaluar las condiciones de la $\mathrm{CE}$, conformado por tres capítulos que se describen en la Tabla 2. La estructura de cada plantilla de evaluación construida por los expertos se presenta en la Tabla 3.

Etapa II: los observadores aplicaron el cuestionario en la $\mathrm{CE}$ de la institución 1 y se obtuvo un índice Kappa de $\mathbf{0 , 7 6}$ (IC 0,69-0,83).

Etapa III: se procedió a continuar en tres CE de diferentes instituciones de la ciudad de Bogotá DC. En la institución 2 se obtuvo un índice Kappa de 0,91 (IC 0,87 - 0,96); en la institución 3 fue $\mathbf{0 , 9 3}$ (IC 0,89 $0,97)$ y en la 4 de $\mathbf{0 , 9 8}$ (IC 0,96 - 100) (Tabla 4).

\section{Discusión}

La CE es uno de los proveedores internos más importantes de insumos medicoquirúrgicos debido al impacto que tiene en el control de las infecciones intrahospitalarias y en la atención de calidad a los pacientes. Por lo tanto, es importante adoptar un sistema de evaluación y verificación que permita realizar auditoría interna para garantizar la calidad en los procesos de esterilización. El cuestionario integra los aspectos referentes a las áreas, métodos de esterilización y garantía de la calidad en centrales. El proceso de evaluación integral de las condiciones de la CE es fundamental, para establecer el cumplimiento correcto de los procesos e induce a tomar medidas para el mejoramiento continuo.
En la aplicación del cuestionario realizada en la CE de la institución 1, se obtuvo un índice Kappa de 0,76, por lo tanto la fuerza de la concordancia fue buena, el acuerdo entre los observadores fue de $91,4 \%$; en la institución 2 se obtuvo un índice de $\mathbf{0 , 9 1}$ con un acuerdo de 96,9\%; en la institución 3 fue de 0,93 con un acuerdo de $97,5 \%$ y en la 4 de 0,98 y un acuerdo final de $99,5 \%$.Con estos valores se puede observar que la fuerza de concordancia que obtuvo al final el cuestionario está descrita en el rango más alto como muy buena.

\section{Conclusiones}

El resultado de este proyecto es un cuestionario que permite evaluar y verificar las condiciones de la CE y puede ser usado por los coordinadores de las centrales para hacer auditoría interna o por evaluadores externos.

\begin{tabular}{|l|l|}
\hline Tabla 1. Escala indice de concordancia Kappa \\
\hline \multicolumn{2}{|c|}{ Fuerza de la concordancia } \\
\hline Valor K & \\
\hline$<0.20$ & Pobre \\
\hline $0.21-0.40$ & Débil \\
\hline $0.41-0.60$ & Moderada \\
\hline $0.61-0.80$ & Buena \\
\hline $0.81-1.00$ & Muy buena \\
\hline
\end{tabular}

\begin{tabular}{|c|c|c|}
\hline \multicolumn{3}{|c|}{ cuestionario } \\
\hline Capitulo I & Capítulo 2 & Capitulo 3 \\
\hline Áreas de la CE & $\begin{array}{l}\text { Métodos de } \\
\text { esterilización }\end{array}$ & Gestión en CE \\
\hline Recepción y lavado & $\begin{array}{l}\text { Calor húmedo y } \\
\text { calor seco }\end{array}$ & Recurso humano \\
\hline Inspección y empaque & Óxido de etileno. & Registros \\
\hline $\begin{array}{l}\text { Preparación de } \\
\text { paquetes y materiales }\end{array}$ & $\begin{array}{l}\text { Plasma de } \\
\text { peróxido de } \\
\text { hidrógeno }\end{array}$ & Guías de proceso \\
\hline Esterilización & $\begin{array}{l}\text { Equipo gas de } \\
\text { formaldehído }\end{array}$ & Trazabilidad \\
\hline $\begin{array}{l}\text { Almacenamiento y } \\
\text { distribución }\end{array}$ & $\begin{array}{l}\text { Ácido peracético } \\
\text { en equipo }\end{array}$ & $\begin{array}{l}\text { Dispositivos } \\
\text { médicos de un solo } \\
\text { uso }\end{array}$ \\
\hline
\end{tabular}




\section{Tabla 3. Plantilla de evaluación}

\begin{tabular}{|c|c|c|c|c|c|}
\hline \multicolumn{6}{|c|}{$\begin{array}{r}\text { Tabla 3. Plantilla de eva } \\
(0,3) \text { CUESTIONARIO PARA EVALUAR LAS CONDICIONES DE LA } \\
\text { RECEPCIÓN Y LAVADO }\end{array}$} \\
\hline & PROCESO & SI & NO & NO APLICA & OBSERVACIONES \\
\hline & DESCRIPCIÓN & 1 & 0 & & \\
\hline 1 & $\begin{array}{l}\text { Se disminuye la carga microbiana de los elementos quirúrgicos con jabón } \\
\text { enzimático en el sitio de uso (Sumergiéndolo, humedeciéndolo con atomizador } \\
\text { o manteneniéndolo con un paño húmedo). }\end{array}$ & & & & \\
\hline 2 & $\begin{array}{l}\text { El instrumental se transporta al área de lavado de la CE en un recipiente } \\
\text { cerrado evitando derrames. }\end{array}$ & & & & \\
\hline 3 & $\begin{array}{l}\text { Se realiza el proceso de recepción y verificación en forma manual o por código } \\
\text { de barras, cualitativa y cuantitativamente. }\end{array}$ & & & & \\
\hline 4 & $\begin{array}{l}\text { Se diligencia el registro de cada equipo o dispositivo médico (con fecha, } \\
\text { nombre de equipo, número de sala, estado completo o incompleto, recibo, } \\
\text { entrega, observaciones). }\end{array}$ & & & & \\
\hline 5 & Se identifican los elementos y su forma de lavado manual o mecánico. & & & & \\
\hline 6 & $\begin{array}{l}\text { Se prepara la solución de detergente enzimatico de acuerdo con las } \\
\text { instrucciones del fabricante. }\end{array}$ & & & & \\
\hline 7 & Se registra la fecha de preparación del detergente enzimático. & & & & \\
\hline 8 & $\begin{array}{l}\text { Lavado manual; los elementos con piezas compuestas se desarticulan y se } \\
\text { sumergen en detergente enzimático, según ficha técnica. }\end{array}$ & & & & \\
\hline 9 & $\begin{array}{l}\text { Se fricciona el instrumental con cepillo de cerdas suaves así como los } \\
\text { elementos canulados. }\end{array}$ & & & & \\
\hline 10 & Se enjuaga cada elemento con abundante agua blanda o desmineralizada. & & & & \\
\hline 11 & Las piezas canuladas se enjuagan con agua blanda a presión. & & & & \\
\hline 12 & $\begin{array}{l}\text { Se seca cada elemento con un paño suave que no suelte motas o con aire } \\
\text { comprinido. }\end{array}$ & & & & \\
\hline
\end{tabular}

\begin{tabular}{|c|c|c|c|c|}
\hline \multicolumn{7}{|c|}{ Tabla 4. Resultados } \\
\hline \multirow{2}{*}{ Institucion } & Valor K & Acuerdo & Error Estándar & Intervalo de confianza (IC) \\
\hline $\mathbf{I}$ & 0,76 & $91,4 \%$ & 0,047 & $(0,69-0,83)$ \\
\hline $\mathbf{2}$ & 0,91 & $96,9 \%$ & 0,048 & $(0,87-0,96)$ \\
\hline $\mathbf{3}$ & 0,93 & $97,5 \%$ & 0,048 & $(0,89-0,97)$ \\
\hline $\mathbf{4}$ & 0,98 & $99,5 \%$ & 0,048 & $(0,96-1,00)$ \\
\hline
\end{tabular}

Según los resultados de este proyecto se puede determinar que la evaluación realizada con este instrumento arroja resultados válidos y confiables sobre las condiciones de la $\mathrm{CE}$, de tal manera que puede determinarse el cumplimiento de acuerdo con estándares preestablecidos, permitiendo la toma de decisiones con cursos de acción apropiados enfocados a mejorar la calidad de los procesos. Por lo tanto, el cuestionario de evaluación responde a las necesidades de los procesos de aseguramiento de la calidad en la prestación de servicios de salud en Colombia, a partir de los lineamientos del sistema único de habilitación y el manual de buenas prácticas en central de esterilización.

\section{Referencias}

1. OPS. Manual de Esterilización para Centros de Salud. Washington: OPS: 2008.

2. Colombia. Ministerio de la Protección Social. Resolución 1043 de 2006 por la cual se establecen las condiciones que deben cumplir los Prestadores de Servicios de Salud para habilitar sus servicios e implementar el componente de auditoría para el mejoramiento de la calidad de la atención y se dictan otras disposiciones. (Diario oficial 46271 de Mayol7 de 2006).

3. Colombia. Ministerio de la Protección Social. Decreto 2309 de 2002, por el cual se expide el Manual de Buenas Prácticas de Esterilización para Prestadores de Servicios de Salud.

4. Colombia. Ministerio de Salud. Anexo Técnico 1. Resolución 1439 de 2002. Por la cual se adoptan los Formularios de Inscripción y de Novedades para el Registro Especial de Prestadores de Servicios de Salud, los Manuales de Estándares y de Procedimientos, y se establecen las Condiciones de Suficiencia Patrimonial y Financiera del Sistema Único de Habilitación de Prestadores de Servicios de Salud y los definidos como tales. (Diario oficial 45000 de noviembre 162002 ).

5. Vaughan P, Morrow Richard. Salud Comunitaria: Manual de epidemiología para la gestión de sistemas locales de salud SILOS. México: Pax; 1997. 